\title{
SIMULATIONS OF SHOCK-TURBULENCE INTERACTIONS: FROM CANONICAL PROBLEMS TO ENGINEERING APPLICATIONS
}

\author{
Sanjiva Lele \\ Professor of Aeronautics and Astronautics and of Mechanical Engineering
}

\begin{abstract}
Many applications in engineering and physical sciences involve situations where a turbulent flow interacts with shock waves. High-speed flows around aerodynamic bodies and through propulsion systems for high-speed flight are bound with interactions of shear-driven turbulence with complex shock waves. Prediction of shockinduced separation, unsteady loads and heat-transfer in such systems is a significant engineering challenge. Numerical simulations of such physical phenomena impose conflicting demands on the numerical algorithms. Capturing broadband spatial and temporal variations in a turbulent flow suggests the use of high-bandwidth schemes with minimal dissipation and dispersion, while capturing a flow discontinuity at a shock wave requires numerical dissipation. Results from DNS of a canonical shock-turbulence interaction problem, i.e. the interaction of isotropic turbulence with a (nominally) normal shock, are discussed first, highlighting the effect of shock strength and turbulence intensity, and contrasted with linear theory where possible. Significant nonlinear effects in the post-shock region are observed and explored. Results from the interaction of a spherical blast wave and a converging shock wave with turbulence will be contrasted with the planar problem. Finally as a bridge from simpler, idealized cases of shock-turbulence interaction to applications in engineering, some highlights from LES of jet injection and mixing in a supersonic crossflow, oblique shock wave interaction with a turbulent boundary layer, supersonic flow in a compression ramp, and shock-induced unsteadiness in an over-expanded nozzle will be discussed. The physical insights enabled by DNS and LES of high-speed compressible turbulent flows will be emphasized and open questions and modeling issues discussed along the side.
\end{abstract}

